\title{
Hydrocolpos with peritonitis in the newborn
}

\author{
I GUPTA* AND AJ BARSON \\ From the Department of Pathology, St Mary's Hospital, Manchester, UK
}

SUMmaRY Two cases of hydrocolpos are described presenting soon after birth. One infant who died on the first day had an intrauterine peritonitis caused by compression of the caecum on the pelvic brim. The other surviving infant developed a caecal perforation secondary to Hirschsprung's disease. Neither infant had rectal atresia, which is the commonest cause of intestinal obstruction in these infants. The association of hydrocolpos with polydactyly in one of these cases may represent an autosomal recessive trait.

Congenital dilatation of the vagina, occasionally also associated with enlargement of the uterine cavity (hydrometrocolpos), occurs in less than 1 in 30000 births. $^{12}$ It requires some form of developmental obstruction of the vaginal tract for its genesis. This is usually represented by a thick vaginal membrane containing muscular tissue in its core or by some degree of vaginal atresia. ${ }^{34}$ Both these conditions are embryologically distinct from imperforate hymen, which is a far less common precursor than was once thought. A necessary additional factor for these patients who present in the neonatal period is an abundant secretion of mucus from the endometrial and endocervical glands, possibly an effect of circulating maternal oestrogen. It usually presents as a large, suprapubic mass, often accompanied by a cystic swelling within the introitus, and by retention of urine and faeces. The original description is probably that given by Godefroy in $1856,{ }^{5}$ but there are now in excess of 100 reports in the literature. 467 In a recent series of 26 cases, 12 were reported to have imperforate anus. ${ }^{7}$ Although this incidence is higher than that previously reported there is no doubt that this is the most frequent cause of intestinal obstruction, demanding surgical intervention. The two cases of hydrocolpos which are the subject of the present paper both had peritonitis, one secondary to direct compression of the caecum by the dilated vagina against the pelvic brim, and the other secondary to rectal Hirschsprung's disease. No similar reports have been found in the literature.

\section{Case reports}

CASE 1

A girl was born to a 25-year-old mother who had *Present address: Department of Pathology, Royal Albert Edward Infirmary, Wigan WN1 2NN.

Received for publication 4 December 1979 had two previous normal pregnancies. The present pregnancy had been complicated by hyperemesis at eight weeks' gestation that required four days' inpatient treatment. She subsequently attended the antenatal clinic regularly and was admitted again at 36 weeks' gestation with mild pre-eclampsia. Her hypertension and proteinuria worsened during the following two weeks, necessitating induction of labour. There was a normal vertex vaginal delivery but the infant was deeply cyanosed and bradycardic and showed no spontaneous respiratory effort. The infant required positive pressure ventilation via an endotracheal tube from birth. A large abdominal mass was noted, which on a plain radiograph appeared to be arising from the pelvis. Urethral catheterisation was not successful, but about $100 \mathrm{ml}$ of turbid yellow fluid was obtained by suprapubic aspiration, and on this account the abdominal mass was thought to be a distended bladder. The infant was transferred to the Regional Neonatal Surgical Unit in St Mary's Hospital and maintained on a Bourne's ventilator. She was completely inactive, assuming a hypotonic frog-like position. Despite $100 \%$ oxygen, there was persistent acidaemia and hypercapnia. Bilateral pneumothoraces developed, but the lungs re-expanded well after drainage. Ventilation was discontinued terminally after further neurological deterioration and profound bradycardia. Death occurred 18 hours after birth.

\section{Necropsy}

The infant weighed $2904 \mathrm{~g}$ and had a crown-heel length of $45.0 \mathrm{~cm}$ and a crown-rump length of 32.1 $\mathrm{cm}$. She had a mongoloid facies with a flat, dimpled nose, crumpled but normally positioned ears, and a short, broad neck. The suprapubic mass noted during life was found to be a grossly dilated uterus and vagina (Figs 1 and 2), measuring together $4 \mathrm{~cm}$ 


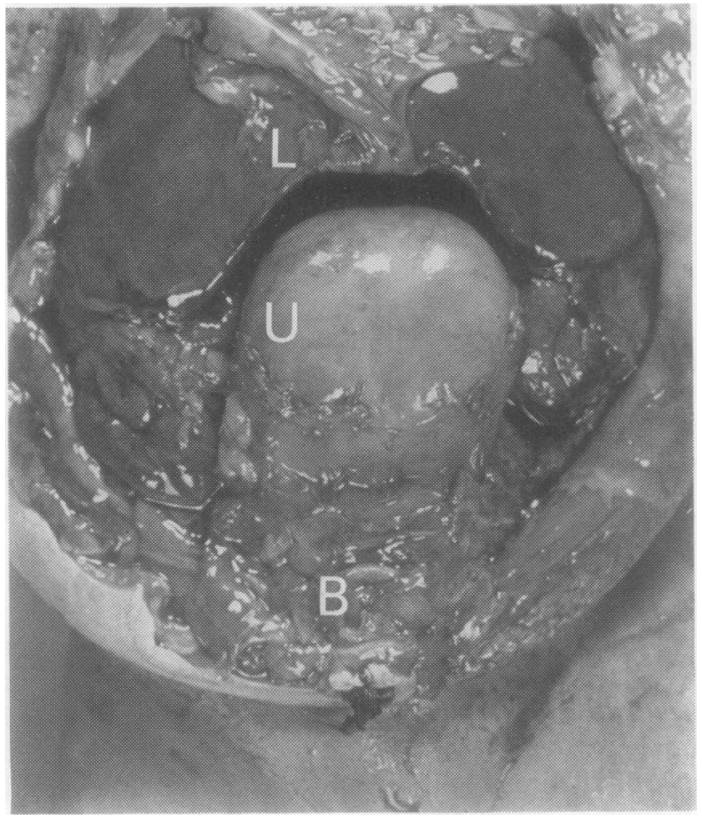

Fig. 1 Case 1. Postmortem appearance after reflection of the abdominal wall. The distended uterus $(U)$ has indented the liver $(L)$. The bladder $(B)$ was empty.

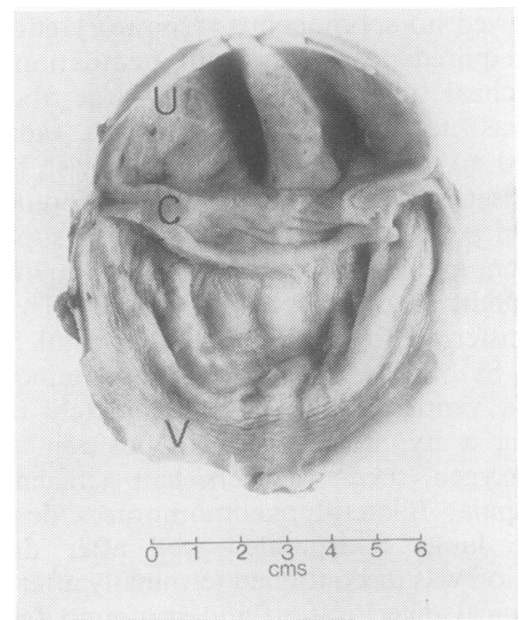

Fig. 2 Case 1. Hydrometrocolpos, opened anteriorly and with the mucoid contents removed: uterus $(U)$; cervix $(C)$; vagina $(V)$.

in width and $8 \mathrm{~cm}$ in length. Both organs were filled with a mucoid opalescent secretion. Ovaries were seen on either side of the cystic vaginal vault with both fallopian tubes adherent to the sides of the distended uterus. No hymen could be identified. The uterus was so large that the liver had a broad notch between the right and left lobes in order to accommodate the uterine fundus (Fig. 1). Over the surface of the hydrometrocolpos and between the coils of intestines were numerous adhesions indicative of a peritonitis. The caecum and appendix were firmly wedged between the brim of the true pelvis and the enlarged uterus. Peritoneal adhesions were particularly dense in this region, and it was felt that the peritonitis had been caused by a caecal perforation secondary to obstruction from compression between the pelvic brim and the large uterus. Histologically, the caecum and terminal ileum were infarcted, and widespread peritonitis appeared to be the result of the extrusion of meconium into the peritoneal cavity. There was a dense fibrous perisplenitis in which focal calcification had occurred. Both kidneys were moderately hydronephrotic, and the left kidney contained a cyst, $1 \mathrm{~cm}$ in diameter, in the upper pole. Both ureters were dilated but the bladder was of normal size and empty.

The occipitofrontal head circumference was $32 \mathrm{~cm}$. There was a marked generalised cerebral oedema with flattening of the gyri, together with coning of the cerebellar vermis for $0.5 \mathrm{~cm}$ below the level of the foramen magnum. Well-developed hyaline membranes were seen lining the alveoli microscopically. No pathogens were isolated from postmortem culture of the blood, lung, peritoneum, or contents of the uterus. Radiologically, the skeleton was normal with a bone maturity of 37 weeks. Double sex chromatin bodies were identified in $5 \%$ of the cells examined from a buccal smear, but postmortem skin fibroblast culture demonstrated a normal female karyotype (46XX), confirmed by banding, with no evidence of mosaicism.

\section{CASE 2}

A girl weighing $2660 \mathrm{~g}$ was delivered at 39 weeks' gestation by elective Caesarean section for cephalopelvic disproportion to a woman of 20 with a normal son. Extra digits were present on both feet and on the left hand. The left foot had six metacarpal bones. The father's uncle had polydactyly. Both parents were normal, Asian in origin, and unrelated. The infant was transferred to the Regional Neonatal Surgical Unit at 32 hours of age because of abdominal distension, vomiting, and failure to pass meconium. A firm mass arising from the pelvis could be palpated, and both legs were congested. There was a small right pneumothorax that subsequently resolved after drainage. Laparotomy on the second day revealed a large hydrocolpos filling most of the abdomen. The large bowel was distended down to the rectosigmoid junction, and it was thought at this time that intestinal obstruction had been caused by compression of the gut on the pelvic brim. Both ovaries were 
identified on either side of a normally sized uterus. There was complete atresia of the lower one-third of the vagina with distension proximally (Fig. 3).

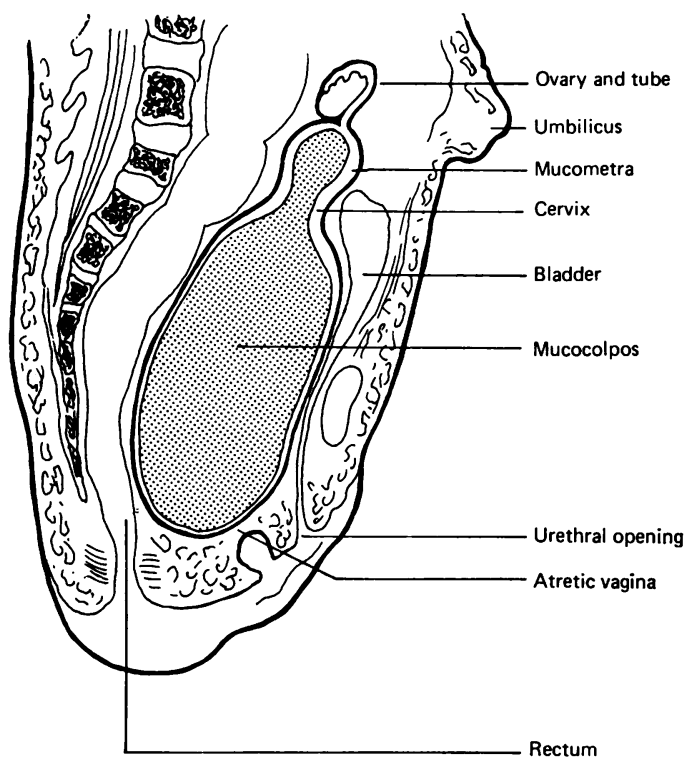

Fig. 3 Case 2. Diagrammatic representation of the anatomy in a saggital plane.

The vagina was opened through the anterior wall, and an anastomotic channel was created with the vulval mucosa. Two days postoperatively a pneumoperitoneum developed. A vaginogram showed the previous surgical incision to be intact, and a diagnosis of intestinal perforation was made. This was confirmed at a second laparotomy on the fourth day of life. The transverse and ascending colon were seen to be very distended, and there was a caecal perforation. A caecostomy and appendicectomy were performed. Biopsies were taken from the caecum and sigmoid colon. Histologically, ganglion cells were identified in both the caecum and appendix, and both showed an acute suppurative peritonitis. The biopsy from the sigmoid colon was devoid of ganglion cells, and there was an excessive proliferation of nerve fibrils characteristic of Hirschprung's disease. The infant made a good postoperative recovery and was allowed home at 5 weeks of age. Examination of the chromosomes demonstrated a normal female karyotype (46XX).

\section{Discussion}

In comparison with hydrocolpos, haematocolpos at adolescence in association with an imperforate hymen is a far more common event. The differences in the pathogenesis of these two conditions are now clearly recognised but still poorly understood. In the earliest descriptions of congenital hydrocolpos, it is likely that what was thought to be an imperforate hymen was in fact a form of vaginal atresia the embryogenesis of which differs fundamentally from that of the hymen. ${ }^{8}$ Although some cases of hydrocolpos have vaginal atresia of a degree which leaves no room for dispute as to its nature, in others the obstruction has the form of a vaginal membrane. As long ago as 1912 Blair Bell $^{9}$ showed that such obstructing membranes occurred at a variable distance above the hymenal fringe. In the older embryological literature ${ }^{10}$ it was thought that only the lower one-fifth of the vagina was derived from the urogenital sinus, and that vaginal membranes represented a failure of canalisation between this and the lower end of the Mullerian uterovaginal canal. It was subsequently shown that the whole of the vaginal epithelium originates from the sinus upgrowth between crown-rump stages $65 \mathrm{~mm}$ to $140 \mathrm{~mm}$ (approximateley 13 to 18 weeks). This sinus upgrowth is initially a bilateral structure. If its medium septum persists, congenital duplication of the vagina may be combined with a double hydrometrocolpos. ${ }^{12}$ The variable level of a congenital vaginal membrane is a reflection of the cranial migration of an occluding plug of Mullerian and sinus cells as the vagina develops in this direction. Histologically, the upper surface of the obstructing membrane should be lined by columnar epithelium and the lower surface by stratified squamous epithelium $^{9}$ but this differentiation may be modified under the influence of maternal oestrogens. ${ }^{3}$ If the upgrowth from the urogenital sinus does not take place, the result is a vaginal atresia rather than a persistent vaginal membrane. Imperforate hymen has a different embryogenesis since cases have been reported in the absence of a vagina. The hymen is thought to represent remnants of a urogenital membrane in a way analogous to that of an imperforate anus. ${ }^{3}$ Some caution must be exercised in acknowledging the commonly accepted association of hydrocolpos with imperforate anus since the term is often used when rectal atresia would be a more accurate description. On embryological grounds it is more likely that the association is between rectal and vaginal atresia.

The clinical features of hydrocolpos in the newborn are dominated by the large size of the distended vagina which is as much the product of secretory activity as it is of obstruction to vaginal outflow. This is generally believed to be a manifestation of circulating maternal oestrogen, which appears to affect the histological differentiation of the genital tract when the fetus has attained a crown-rump 
length of $112 \mathrm{~mm}$ (approximately 16 weeks).11 The phenomenon is similar to infantile gynaecomastia, and the transient nature of the effect is supported by the occasional clinical experience of a vaginal obstruction resealing itself after an initial drainage procedure. ${ }^{4}$ Little vaginal and uterine mucus reaccumulates after an early postnatal evacuation.

The nature of the vaginal obstruction in case 1 was not identified, almost certainly because it was disrupted in the process of the unsuccessful catheterisation of the bladder, in the mistaken belief that this was the cause of the abdominal mass, an eventuality which has been experienced by others. ${ }^{4}$ Catheterisation is made difficult because the distended vagina tends to retract the urethral orifice into the introitus. This case showed a hugely distended uterine cavity (Fig. 2) and is therefore correctly termed a hydrometrocolpos. However, most cases of hydrocolpos do not involve the uterus to the same degree, and case 2 is more typical in this respect. Major urinary difficulty is common and occurred in about $70 \%$ of a series of 85 cases referred to by Dajani. ${ }^{6}$ In another series of 26 infants, radiographic studies demonstrated hydronephrosis, usually bilateral, in 10.7 Renal hypoplasia, agenesis, and polycystic kidney have also been reported.

A frequent complication of hydrocolpos is gastrointestinal obstruction. In a series of 22 cases reviewed by Dooley, ${ }^{13}$ four were said to have imperforate anus, while in the 26 infants described by Reed and Griscom ${ }^{7} 12$ had imperforate anus and one severe anal stenosis. In addition, there are cases referred to in the literature in which intestinal obstruction has been caused by simple compression rather than by intestinal malformation. While these may be cured by drainage of the hydrocolpos there are occasional cases where the dense mass of peritoneal adhesions seen at laparotomy in the newborn indicate an established intrauterine peritonitis. Such infants have a poor prognosis, ${ }^{14}$ and case 1 comes within this category. The presence of a meconium peritonitis was confirmed histologically together with the calcification of soft tissues in response to this. Occasionally, this is detectable on an abdominal radiograph. ${ }^{\text {? }}$ In the absence of malformation of the intestine, previous reports of peritonitis with hydrocolpos have not described the mechanism of the obstruction. In case 1 , this was clearly due to compression of the caecum on the pelvic brim by the distended uterus and vagina, a mechanism which probably also accounts for the hydroureter and hydronephrosis which were also present. In case 2, the peritonitis was postnatal in origin, but no other case could be found in the literature where this was precipitated by Hirschsprung's disease. The narrowed aganglionic rectum seen at the initial laparotomy was mistaken for the effect of compression of the rectosigmoid junction on the pelvic brim. In this infant there was also a complete atresia of the lower one-third of the vagina.

Polydactyly has been observed in a proportion of several series of infants with hydrocolpos. ${ }^{1713}$ Some cases at least of hydrocolpos with polydactyly appear to be inherited as an autosomal recessive trait, ${ }^{15-18}$ and there is an association also with congenital heart disease ${ }^{15} 18$ and Ellis-van-Creveld syndrome. ${ }^{17}$ Case 2 might come into this category although neither case has to date a familial history. Both had normal karyotypes on fibroblast culture. Case 1 had double sex chromatin bodies in a small proportion of cells examined from a buccal smear.

Finally, the importance of making a correct diagnosis should be stressed. Apart from distension of the bladder, hydrocolpos has been confused with ovarian, urachal, and mesenteric cysts, anterior meningocele, and teratoma. In one series of 40 cases, six infants unnecessarily had the uterus and vagina removed, ${ }^{4}$ and similar reports exist elsewhere in the literature. ${ }^{161920}$ In the absence of vaginal atresia, a simple incision of the obstructing membrane suffices to correct the condition. The presence of a cystic mass within the vulva, which enlarges when the infant strains, is an invaluable clinical sign but is present in only about half the reported cases. ${ }^{13}$ Radiographic studies, including the injection of radio-opaque medium into the mass, can often establish the diagnosis where doubt exists.?

Our thanks are due to Mr SJ Cohen and Miss CM Doig for access to their clinical records.

\section{References}

1 Westerhout FC, Jr, Hodgman JE, Anderson GV, Sack RA. Congenital hydrocolpos. Am J Obstet Gynaec 1964;89:957-61.

${ }^{2}$ Ho NK. Hydrocolpos. Aust NZ J Obstet Gynaec 1975; 15:186-8.

${ }^{3}$ Kanagasuntheram R, Dassanayake AGS. Nature of the obstructing membrane in primary cryptomenorrhoea. J Obstet Gynaec Brit Emp 1958;65:487-92.

4 Spence HM. Congenital hydrocolpos. A review with emphasis on urologic aspects and a report of four additional cases. JAMA 1962;180:1100-5.

${ }^{5}$ Godefroy M. Imperforation de la membrane hymen produisant des accidents chez une enfant de deux mois. Opération. Guérison. Lancette Fr, Gaz Hôp Civ Milit 1856;29:567.

- Dajani AM. Hydrocolpos: a cause of urinary obstruction. Int Surg 1972;57:848-50.

${ }^{7}$ Reed MH, Griscom NT. Hydrometrocolpos in infancy. Am J Roentgenol Radium Ther Nucl Med 1973; 118:1-13. 
${ }^{8}$ Mahoney PJ, Chamberlain JW. Hydrometrocolpos in infancy-congenital atresia of the vagina with abnormally abundant cervical secretions. $J$ Pediatr 1940;17:772-80.

9 Blair Bell W. Further investigations into the chemical composition of menstrual fluid and the secretions of the vagina, as estimated from an analysis of haematocolpos fluid, together with a discussion of the clinical features associated with haematocolpos, and a description of the character of the obstructing membrane. J Obstet Gynaec Brit Emp 1912;21:209-15.

${ }^{10}$ Koff AK. Development of the vagina in the human fetus. Contr Embryol Carnegie Instn 1933;24:61-90.

${ }^{11}$ Bulmer $\mathrm{D}$. The development of the human vagina. J Anat 1957;91:490-509.

${ }^{12}$ Davis C, Jr, Fell EH. Double hydrometrocolpos and imperforate anus in a newborn infant. Am $\mathrm{J}$ Dis Child 1950;80:79-84.

${ }^{13}$ Dooley RT. Hydrometrocolpos. Report of a case in a newborn infant. Am J Dis Child 1962;103:692-6.

${ }^{14}$ Dennison WM, Bacsich P. Imperforate vagina in the newborn. Neonatal hydrocolpos. Arch Dis Child $1961 ; 36: 156-60$.
${ }^{15}$ McKusick VA, Bauer RL, Koop CE, Scott RB. Hydrometrocolpos as a simply inherited malformation. JAMA 1964;189:813-6.

${ }^{16}$ McKusick VA, Weilbaecher RG, Gragg GW. Recessive inheritance of a congenital malformation syndrome. JAMA 1968;204:113-8.

${ }^{17}$ Dungy CI, Aptekar RG, Cann HM. Hereditary hydrometrocolpos with polydactyly in infancy. Pediatrics 1971;47:138-41.

${ }^{18}$ Kaufmann RL, Hartmann AF, McAlister WH. Family studies in congenital heart disease, II: A syndrome of hydrometrocolpos postaxial polydactyly and congenital heart disease. (Clinical Delineation of Birth Defects. XV Cardiovascular System.) Birth Defects Orig Art Ser 1972;8:85-7.

${ }^{19}$ Cook GT, Marshall VF. Hydrocolpos causing urinary obstruction. J Urol 1964;92:127-32.

20 Joseph MK, Nayar BT, Kannankutty M. Hydrohaematocolpos. Br Med J 1966;1:89.

Requests for reprints to: Dr AJ Barson, Department of Pathology, St Mary's Hospital, Whitworth Park, Manchester M13 0JH, UK. 